\title{
Síntese de membranas de fibra oca de Polisulfona/Alumina para o tratamento de água na presença do corante índigo blue
}

\author{
Synthesis of Polysulfone / Alumina hollow fiber membranes for water treatment in the presence of \\ indigo blue dye
}

Síntesis de membranas de fibra hueca de polisulfona / alúmina para tratamiento de agua en presencia de colorante azul índigo

\section{Resumo}

Motivado pelas necessidades tecnológicas e ambientais, os processos de separação por membranas têm exibido uma grande melhora em relação às técnicas tradicionais. Estes benefícios incentivam a aplicação de separações por membranas e estimulam o desenvolvimento em processos de purificação e esterilização nas indústrias farmacêutica e alimentícia, em geral. Além disso, elas são utilizadas no tratamento de água para uso industrial ou urbano, dessalinização, separação de gases, clarificação de sucos, hemodiálise e no tratamento de efluentes. Esta última, é a aplicação que este trabalho se destina, especialmente na separação de água dos corantes têxteis, isto é, o índigo blue. Dessa forma, foram sintetizadas membranas de polisulfona na forma de fibra oca, com teores variados de alumina, para uso no tratamento de efluentes. Foram avaliadas as características morfológicas, a hidrofilicidade, as medidas de fluxo e a medida de turbidez delas. As membranas foram produzidas pelo método da inversão de fase, via extrusão a frio, imersão - precipitação. Os resultados obtidos por filtração indicam que as partículas de alumina podem aumentar o fluxo de água melhorando a hidrofilicidade da membrana. Por MEV, foi verificada uma maior quantidade de poros, de tamanhos menores, com a inserção da alumina na polisulfona. Desse modo, por meio da análise do Turbidímetro, a quantidade de partículas suspensas de índigo blue na membrana com alumina foi menor, visto que os poros destas são menores e em maior quantidade, gerando uma maior remoção do corante. Assim, com o aumento do teor de alumina, foi melhorada a separação entre a água e o corante índigo blue.

Palavras-chave: Alumina; Polisulfona; Índigo blue; Tratamento de efluentes.

\begin{abstract}
Motivated by technological and environmental needs, membrane separation processes have shown a great improvement over traditional techniques. These benefits encourage the application of membrane separations and stimulate development in purification and sterilization processes in the pharmaceutical and food industries in general. In addition, they are used in the water treatment for industrial or urban use, desalination, gas separation, clarification of juices, hemodialysis and in the effluents treatment. The latter is the application that this work is intended for,
\end{abstract}


especially in the separation of water from textile dyes, i.e., indigo blue. Thus, polysulfone membranes were synthesized in the form of hollow fiber, with varying levels of alumina, for use in the effluents treatment. The morphological characteristics, hydrophilicity, flow measurements and the turbidity measurement of the prepared membranes were evaluated. The membranes were produced by the phase inversion method, via cold extrusion, immersion - precipitation. Filtration experiments indicate that alumina particles can increase the flow of water by improving the hydrophilicity of the membrane. By SEM images, a larger amount of pores, of smaller sizes, was verified with the insertion of alumina in the polysulfone. Thus, through the analysis of the Turbidimeter, the amount of indigo blue particles suspended in the membrane with alumina was smaller, since the pores are smaller and their greater quantity, generating a greater removal of the dye. Thus, with the increase in the alumina content, the separation between water and the indigo blue dye was improved.

Keywords: Alumina; Polysulfone; Indigo blue; Effluents treatment.

\section{Resumen}

Motivados por necesidades tecnológicas y medioambientales, los procesos de separación por membranas han mostrado una gran mejora con respecto a las técnicas tradicionales. Estos beneficios fomentan la aplicación de separaciones por membranas y estimulan el desarrollo de procesos de purificación y esterilización en la industria farmacéutica y alimentaria en general. Además, se utilizan en el tratamiento de aguas para uso industrial o urbano, desalación, separación de gases, clarificación de jugos, hemodiálisis y en el tratamiento de efluentes. Esta última es la aplicación a la que está destinado este trabajo, especialmente en la separación de agua de tintes textiles, es decir, azul índigo. Así, se sintetizaron membranas de polisulfona en forma de fibra hueca, con niveles variables de alúmina, para su uso en el tratamiento de efluentes. Se evaluaron sus características morfológicas, hidrofilia, medidas de flujo y medidas de turbidez. Las membranas fueron producidas por el método de inversión de fase, vía extrusión en frío, inmersión - precipitación. Los resultados obtenidos por filtración indican que las partículas de alúmina pueden incrementar el flujo de agua mejorando la hidrofilia de la membrana. Por SEM se verificó una mayor cantidad de poros, de menor tamaño, con la inserción de alúmina en la polisulfona. Así, a través del análisis del Turbidímetro, la cantidad de partículas de azul índigo suspendidas en la membrana con alúmina fue menor, ya que los poros de estas son más pequeños y en mayor cantidad, generando una mayor remoción del tinte. Así, con el aumento del contenido de alúmina, se mejoró la separación entre el agua y el colorante azul índigo.

Palabras clave: Alúmina; Polisulfona; Azul índigo; Tratamiento de aguas residuales.

\section{Introdução}

O planeta vem sofrendo com inúmeras formas de poluição, e ao longo do tempo essa poluição é a causa de enorme desequilíbrio. Dentre os tipos de poluição, a hídrica é uma das piores, visto que a maioria dos materiais e substâncias que são descartados de maneira inadequada atinge os rios, lagos e o mares; afetando a saúde humana, os seres vivos e todo o ecossistema. Uma das substâncias que mais tem poluído as águas é um corante têxtil insolúvel em água conhecido como Índigo blue (Meksi, Kechida, \& Mhenni, 2007). Dessa forma, a utilização de uma técnica para purificação da água é de suma importância. A aplicação de membranas para filtração e purificação das águas é uma das melhores técnicas da atualidade (Buruga et al., 2019). Esta técnica vem desde a década de 1970, quando surge procedimentos que aplicam membranas sintéticas como barreira seletiva (Habert, Borges, \& Nobrega, 2006). Os processos de separação por membranas (PSM) estão sendo difundidos em diversos campos na indústria. Além disso, possui muitas aplicações na ciência devido às suas vantagens, dentre essas: dimensões relativamente pequenas, operação fácil, menor consumo de energia, design compacto das membranas, baixa pressão operacional, eficiência na remoção da turbidez, patógenos e matéria orgânica. Processos como microfiltração (MF) e ultrafiltração (UF) são utilizados para separação por membrana de baixa pressão. A ciência das membranas tem sido utilizada extensamente para o tratamento de água e esgoto, como é o caso deste trabalho em que serão utilizadas para o tratamento de efluentes têxteis. Para as membranas serem consideradas ideais, são necessárias: alta permeabilidade, resistência mecânica suficiente e excelente estabilidade química (Mulder, 1991; Nakatsuka, Nakate, \& Miyano, 1996; Rodrigues, Mierzwa, \& Vecitis, 2019; Turken, Sengur-Tasdemir, Ates-Genceli, Tarabara, \& Koyuncu, 2019).

Embora as membranas poliméricas tenham uma grande utilidade, apresentam limitações, tais como: durabilidade, resistência a altas temperaturas, facilidade de limpeza, entre outras. Dessa forma, propor um polímero para a fabricação das membranas não é uma escolha aleatória. Usualmente, a escolha é feita com base nas propriedades específicas do polímero. 
Dentre os polímeros conhecidos, podem-se destacar: polisulfona (PSU), politetrafluoretileno (PTFE), poliamidas, poli(acetato de celulose), poli(éter sulfona), poliacrilonitrila (PAN), polieterimida (PEI) e policarbonato (PC) (Xu, Goh, Wang, \& Bae, 2019).

Neste trabalho o polímero escolhido foi a polisulfona, que possui caráter hidrofóbico, boa estabilidade mecânica, térmica e química. É um dos plásticos de engenharia mais conhecidos e utilizados para o desenvolvimento de membranas (Ahn, Chung, Pinnau, \& Guiver, 2008; Yuan, Liu, Liu, \& Wang, 2017). Em membranas, a utilização desse polímero é de grande interesse para aplicações de tratamento de efluentes, separação de gás, nanofiltração (NF), osmose reversa, aplicações médicas, industriais e ultrafiltração (UF) (Anadão, Sato, Wiebeck, \& Valenzuela-Díaz, 2010; Botvay, Máthé, Pöppl, Rohonczy, \& Kubatovics, 1999).

Além do polímero que é um material orgânico, materiais inorgânicos também são utilizados para a produção de membranas. Em contraste com as membranas poliméricas, as membranas cerâmicas possuem boa estabilidade do processo, baixo tratamento preliminar e mínima necessidade de manutenção. Além disso, dispõe de estabilidade química, térmica, mecânica, capacidade de esterilização a vapor, lavagem reversa e alta resistência à abrasão. Também apresentam boas propriedades de separação, extensa vida útil e são bioinertes. Dessa forma, são materiais excelentes para numerosas aplicações na indústria ou no processamento de água e efluentes (LENNTECH, 2019). Com todas essas propriedades das membranas cerâmicas, alguns óxidos metálicos têm sido bastante utilizados, tais como: Óxido de titânio, zircônia, alumina, entre outros. O óxido de alumínio (Al2O3) tratado na ciência dos materiais como alumina, tem estimulado interesse em tecnologia de membranas por pesquisadores, com o intuito de trazer melhorias em membranas já existentes e na criação de novas membranas com características diferentes (Bertotto, Duarte, Santos, Zeni, \& Bergmann, 2018).

Tendo em vista um material que tivesse boas propriedades, são incorporados aos polímeros aditivos. Os polímeros preenchidos com cargas são chamados de compósitos. Essas partículas adicionadas têm o intuito de melhorar propriedades, como: rigidez, hidrofilicidade, diminuir a incrustação, além de aumentar propriedades de barreira, aumentar sua resistência ao fogo e a ignição ou simplesmente reduzir custos. (Alexandre \& Dubois, 2000). Assim, a inserção da alumina no polímero almeja aumentar a hidrofilicidade, aumentar a resistência e também melhorar propriedades de barreira.

Dessa forma, inúmeras pesquisas já estão sendo realizadas utilizando materiais poliméricos com materiais inorgânicos. Diferentes materiais têm sido utilizados, tais como: polímeros com argila (Buruga et al., 2019); polietersulfona e nanotubos de carbono (Vatanpour, Madaeni, Moradian, Zinadini, \& Astinchap, 2011). A polisulfona tem sido utilizada com um variado número de materiais, incluindo alguns tipos de argilas (Rodrigues et al., 2019); a argila montmorilonita (Anadão et al., 2010); TiO2 (Bidsorkhi et al., 2016); nanodiamante silanizado (Tizchang, Jafarzadeh, Yegani, \& Khakpour, 2019) e com a alumina (Homayoonfal, Mehrnia, Rahmani, \& Mohades Mojtahedi, 2015). Neste último caso, ainda há muito a ser estudado e experimentado, para que se possa obter membranas de fibra oca com propriedades que garantam boas aplicações, em especial no tratamento de efluentes.

O objetivo deste estudo foi sintetizar membranas de polisulfona na forma de fibra oca, com teores variados de alumina, a partir do método de inversão de fase, para uso no tratamento de efluentes. Foram avaliadas as características morfológicas, hidrofilicidade, medidas de fluxo e a medida de turbidez das membranas preparadas.

\section{Metodologia}

\subsection{Materiais}

Para esta pesquisa, foram utilizados os seguintes materiais: a polisulfona UDEL P3500 LCD MB7 com massa molar entre $77.000 \mathrm{~g} / \mathrm{mol}$ e $83.000 \mathrm{~g} / \mathrm{mol}$ e densidade relativa de 1.2, segundo ASTMD 792. Adquirida pela Solvay. A alumina 
calcinada (A12O3) - adquirida pela Imerys Fused Minerals. O solvente 1-Metil 2-Pirrolidona (NMP) com 99,5\% de pureza foi utilizado para dissolver o polímero, adquirido da Synth. O polímero polivinilpirrolidona (PVP) foi utilizado como viscosificante, adquirido pela Dinâmica Química Contemporânea Ltda. A Glicerina P.A(Glicerol C3H5(OH)3) - com grau de pureza de 99,5\%, massa molar 92,09 g/mol, adquirido pela Vetec Química Fina Ltda., foi utilizada juntamente com a água para formar uma solução, onde as membranas foram armazenadas para evitar o colapso dos poros.

\subsection{Metodologia}

\section{Preparação das Amostras}

A polisulfona foi seca em estufa a $80^{\circ} \mathrm{C}$ por 24 horas para eliminação da água adsorvida. Em seguida, foi preparada a dispersão da polisulfona e da alumina nas proporções preestabelecidas no estudo. A composição para todas as membranas está apresentada na Tabela 1.

Tabela 1 - Quantidade das composições que foram utilizadas.

\begin{tabular}{|c|c|c|c|c|}
\hline \multirow[t]{2}{*}{ AMOSTRA } & \multirow{2}{*}{$\begin{array}{c}\text { SOLVENTE } \\
(\%)\end{array}$} & \multicolumn{2}{|c|}{$\begin{array}{c}\text { COMPONENTES SÓLIDOS = } \\
\text { POLISULFONA + ALUMINA }(15 \%)\end{array}$} & \multirow{2}{*}{$\begin{array}{c}\text { ADITIVO } \\
\text { PVP } \\
(\%)\end{array}$} \\
\hline & & POLISULFONA (\%) & ALUMINA (\%) & \\
\hline PSU Pura & 80 & 100 & - & 5 \\
\hline $\mathrm{PSU}+3 \% \mathrm{Al}_{2} \mathrm{O}_{3}$ & 80 & 97 & 3 & 5 \\
\hline $\mathrm{PSU}+5 \% \mathrm{Al}_{2} \mathrm{O}_{3}$ & 80 & 95 & 5 & 5 \\
\hline
\end{tabular}

Fonte: Autores.

A solução ficou sob agitação durante o período de $2 \mathrm{~h}$, a uma velocidade de $1200 \mathrm{rpm}$. Esse processo foi repetido para as diferentes composições. Quando finalizado o preparo das soluções, a mesma ficou em repouso por um período de $24 \mathrm{~h}$, para a eliminação de bolhas e estabilização da solução.

Para a produção das membranas do tipo fibra oca, foram utilizados em torno de $400 \mathrm{~g}$ de solução para que se tenha um fluxo continuo durante o processo de fiação. Além disso, ocorreu a preparação utilizando uma extrusora contendo dois canais concêntricos, permitindo a extrusão simultânea do líquido interno e da solução polimérica. Após uma distância de 5cm, a solução polimérica passa da extrusora e entra em contato com o banho de não-solvente (água destilada), onde a mesma permanecerá até completar a precipitação. Em seguida, as fibras foram colocadas em água destilada, por 24h, para a eliminação do solvente residual. Logo depois, as membranas foram colocadas em uma solução contendo $90 \%$ de água e $10 \%$ de glicerina com o objetivo de evitar o colapso dos poros. Além disso, com a finalidade de realizar o ângulo de contato, que não é possível de ser realizado nas fibras, houve a obtenção das membranas planas para cada composição em estudo.

\subsection{Caracterizações das amostras}

\section{Microscopia Eletrônica de Varredura (MEV)}

As fotomicrografias das membranas foram realizadas com objetivo de verificar suas morfologias. Sendo executadas por microscopia eletrônica de varredura (MEV), utilizando o equipamento de marca SHIMADZU, modelo SSX-550 Superscan. As amostras foram submetidas à fratura com nitrogênio líquido com o objetivo de evitar a deformação plástica das 
mesmas. Além disso, as superfícies das amostras são revestidas com ouro com a finalidade de evitar o acúmulo de carga negativa. Foram analisadas a superfície de topo e a secção transversal das membranas.

\section{Ângulo de contato}

A análise do ângulo de contato foi utilizada para determinar a hidrofilicidade das membranas, pela técnica da gota séssil, por meio de um ângulo de contato portátil, modelo Phoenix-i da Suface Eletro Optics - SEO. Fazendo o uso de um dosador micrométrico a gota é depositada sobre a membrana. Dessa forma, 30 imagens foram captadas e analisadas, utilizando um intervalo de 10 segundos, no software do equipamento. A análise foi efetuada a partir das membranas planas.

\section{Medidas de Fluxo}

Para a realização dos ensaios de medidas de fluxo das membranas de fibra oca foram produzidos módulos contendo cada um, 5 membranas. Para a fabricação do módulo foi utilizada uma pipeta de $20 \mathrm{~cm}$. As membranas foram impermeabilizadas para realização da análise com água destilada. Para o sistema de coleta foi utilizado um reservatório para a água, uma bomba centrífuga e um manômetro para medir a pressão do fluxo de água no sistema. A corrente de alimentação com concentração do soluto (CF) é alimentada em escoamento tangencial ao longo da membrana e divide-se em duas correntes, o concentrado ou retido (CR) e o permeado (CP). As membranas foram submetidas as pressões de 1 e 2 bar para o teste de permeabilidade. Durante a filtração, o fluxo foi calculado usando a seguinte equação:

$$
I=\frac{\text { Volume do permeado }}{\text { Tempo } x \text { Ârea da membrana }}
$$

\section{Análise do permeado}

A análise do permeado é realizada utilizando o Turbidímetro, com o objetivo de medir a turbidez da água, que é causada por inúmeros tipos de impurezas suspensas. Foi utilizado um Turbidímetro microprocessado digital, modelo DLTWV, fabricado de acordo com as normas técnicas nacionais e internacionais. A unidade de medida da turbidez da água é a NTU (Unidade Nefelométrica De Turbidez).

\section{Resultados e Discussão}

\subsection{Microscopia Eletrônica de Varredura (MEV)}

Com a finalidade de entender a influência da incorporação de alumina na estrutura final da membrana de polisulfona, a seção transversal das membranas de polisulfona/alumina, foram observadas por microscopia eletrônica de varredura (MEV). As imagens da seção transversal de membranas preparadas a partir de 0,3 e $5 \%$ em peso de alumina são apresentadas na Figura 2. Pode-se observar que, a partir da adição de partículas de alumina na proporção de 3 e $5 \%$, principalmente na proporção de 5\%, mais poros com diâmetros menores foram criados na seção transversal da membrana em comparação com a membrana de polisulfona pura. Além disso, as membranas possuem uma estrutura assimétrica típica com uma camada superior densa e macrovazios totalmente desenvolvidos na parte inferior (Jamshidi Gohari, Lau, Matsuura, \& Ismail, 2013; Vatanpour et al., 2011). É importante ressaltar que os macrovazios no interior reduziram de tamanho com a adição da alumina. Adicionando partículas à matriz da membrana, a morfologia é efetivamente modificada. Isso se deve por conta da inserção da alumina hidrofílica em um polímero hidrofóbico, que resulta em instabilidade termodinâmica na solução. Portanto, a adição de cargas hidrofílicas no polímero atraiu o não solvente para o polímero, o que aumentou a transferência de massa entre o 
solvente e o não solvente, resultando na formação de uma estrutura maior de poros da membrana na sua subcamada (Bidsorkhi et al., 2016; Maximous, Nakhla, Wan, \& Wong, 2009).

Figura 2 - Imagens das micrografias da seção transversal das membranas compósitas polisufona/alumina (numerada com 1 para seção transversal) e (numerada com 2 para ampliação da região do suporte poroso) com diferentes composições: (a) membranas de polisulfona pura; (b) membranas de polisulfona com 3\% de alumina; (c) membranas de polisulfona com 5\% alumina.
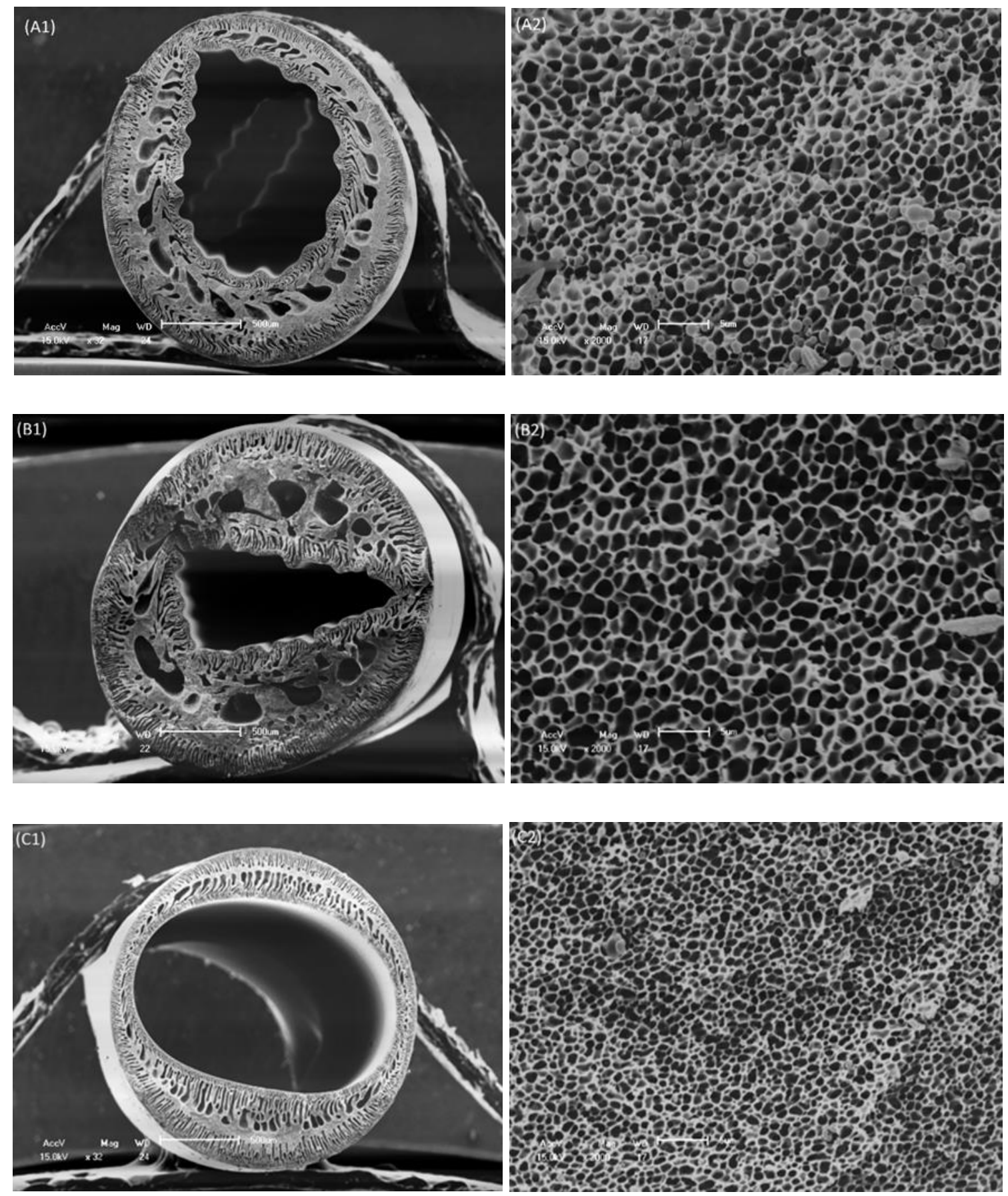

Fonte: Autores. 


\section{2 Ângulo de Contato}

Uma operação frequente para qualificar a hidrofobicidade da membrana é a medida do ângulo de contato com líquidos da sonda (Chen et al., 2016). O método mais utilizado (gota séssil) é uma medida direta do $\theta$, onde uma gota líquida é depositada em uma superfície. O ângulo é determinado através da construção de uma tangente ao perfil no ponto de contato da gota com a superfície sólida. Isso pode ser feito em uma imagem projetada ou em uma fotografia da gota, utilizando um goniômetro acoplado a um computador (Palacio et al., 1999). Sendo assim, quanto maior o ângulo de contato entre a gota e o sólido em que ela está depositada menor será a hidrofilicidade (Vatanpour et al., 2011). Os valores do ângulo de contato nas membranas foram analisados usando a água na medição, para assim verificar a hidrofilicidade das membranas. Como pode ser visto na Figura 3, o gráfico ilustra a diminuição do ângulo de contato com o aumento da alumina, e na Tabela 2 é visto os valores exatos dos ângulos. Dessa maneira, pode existir uma correlação da quantidade de alumina com a hidrofilicidade da membrana, visto que quanto maior a quantidade de alumina no compósito menor é o ângulo de contato, e maior é a hidrofilicidade (Santos Filho et al., 2019). Sendo assim, a inserção da alumina nas membranas ocasionou uma melhora na hidrofilicidade, o que pode gerar uma melhora na eficiência com relação ao fluxo destas membranas.

Figura 3 - Ângulo de contato das membranas de polisulfona com 3 e $5 \%$ de alumina.



Fonte: Autores.

Tabela 2 - Ângulo de contato das amostras.

\begin{tabular}{cc}
\hline Amostras & $\left(^{\circ}\right)$ \\
\hline PSU Pura & 59,37 \\
$\mathrm{PSU}+3 \% \mathrm{Al}_{2} \mathrm{O}_{3}$ & 22,14 \\
$\mathrm{PSU}+5 \% \mathrm{Al}_{2} \mathrm{O}_{3}$ & 17,43 \\
\hline
\end{tabular}

Fonte: Autores.

\subsection{Medidas de Fluxo}

As medidas de fluxo foram realizadas a uma pressão de 1 bar, com água. As membranas de polisulfona/alumina, em seus ensaios de filtração, indicam que as partículas de alumina podem aumentar o fluxo de água com o aumento da hidrofilicidade 
da membrana, mantendo a eficiência da separação através da diminuição da porosidade (Yan, Li, \& Xiang, 2005). Os resultados indicaram que a presença de partículas de alumina na solução, alterou o processo de inversão de fase, alterando a microestrutura e a porosidade da membrana e influenciando o comportamento de filtração da membrana (Homayoonfal et al., 2015). Pode-se perceber esse comportamento através da Figura 4, onde as membranas contendo alumina possuem um maior fluxo em comparação com as membranas de polisulfona pura, especialmente a membrana contendo $3 \%$ de alumina que obteve um fluxo bem superior às outras. Esse fato pode ter ocorrido devido ao aumento do tamanho dos poros, e dos macrovazios. Todavia, é possível comprovar também um maior fluxo inicial das membranas com 5\% em relação as membranas puras, pois embora o tamanho dos poros tenha diminuído a quantidade deles aumentou. Além disso, esse aumento do fluxo das membranas com alumina, pode ser explicado através da análise de ângulo de contato onde foi visto que as membranas de polisulfona/alumina eram mais hidrofílicas. Outrossim, há uma tendência ao decaimento do fluxo em todas as membranas que pode ter ocorrido devido à hidratação ou à incrustação.

Figura 4 - Medidas de fluxo com água para as membranas de polisulfona pura e com 3 e $5 \%$ de alumina.

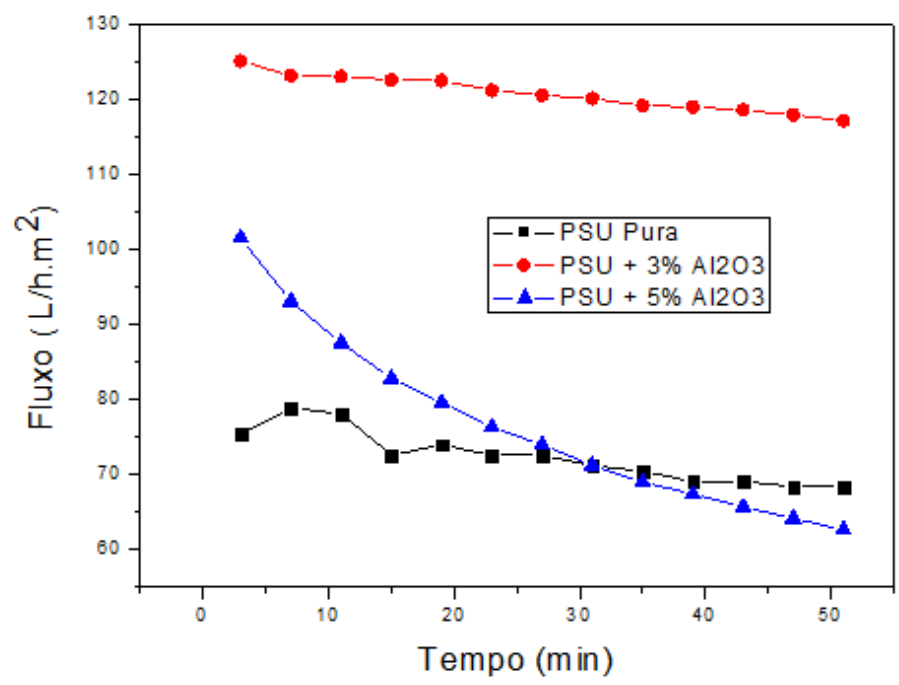

Fonte: Autores.

$\mathrm{Na}$ Figura 5 percebe-se um comportamento contrário ao da Figura 4, isso ocorre por conta que para estas medidas continha uma solução com o corante índigo blue. Nesta situação, as membranas contendo alumina possuem uma tendência a aumentar a quantidade de poros e diminuir o seu tamanho com o aumento da alumina, como visto nas imagens do MEV com a membrana de 5\% de alumina. Dessa forma, as partículas do corante podem ter sido retidas na membrana contendo a alumina, ocasionando uma maior dificuldade no fluxo. Ou seja, o fenômeno da incrustação pode ter ocorrido principalmente nas membranas com alumina. Isso pode ser percebido pois a membrana pura obtém um fluxo maior e as membranas com alumina obtiveram fluxos menores principalmente a membrana com 5\% de alumina (Barbosa Ferreira et al., 2019). No entanto, mesmo com um fluxo menor estas membranas com alumina mostraram melhorias em sua eficiência, no que se refere à quantidade de partículas do corante retidas. A retenção de partículas é vista na análise do permeado. 
Figura 5 - Medidas de fluxo das membranas de polisulfona com 3 e 5\% de alumina, realizadas com água contendo o corante índigo blue.

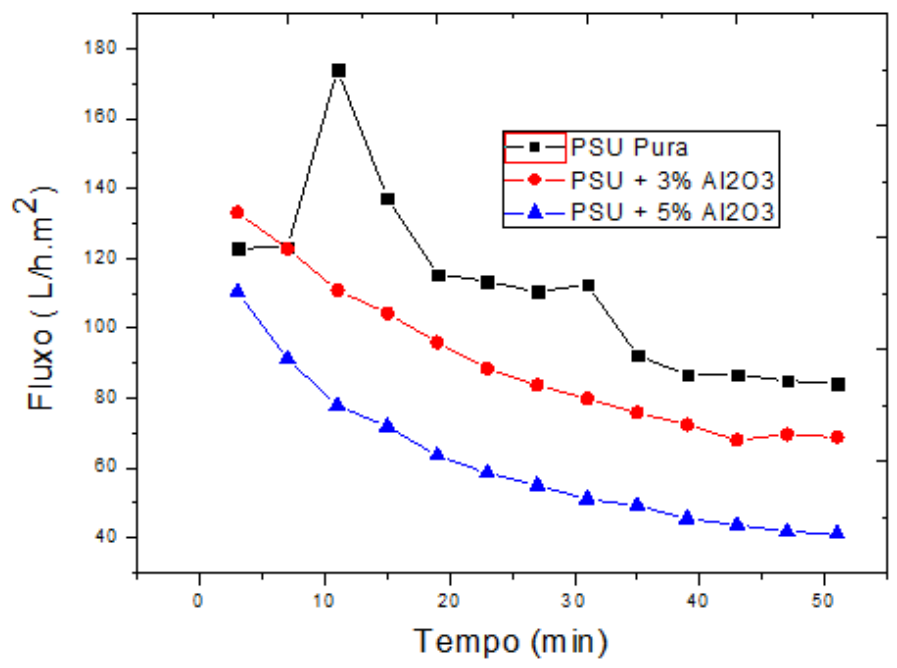

Fonte: Autores.

\subsection{Análise de Turbidez}

Através desta análise é possível verificar a quantidade de partículas presentes na solução antes e após a sua filtração utilizando as membranas. Deste modo, na Tabela 3 estão apresentados os resultados obtidos para esta análise. Por meio dela, foi possível verificar a quantidade de partículas e o coeficiente de rejeição, para as membranas de polisulfona pura e com concentrações de 3 e $5 \%$ de alumina. A solução contendo o índigo Blue foi preparada contendo 300 ppm. Com relação à sua eficiência, percebe-se que as membranas, em especial aquelas que contem alumina, apresentam uma alta rejeição ao corante índigo Blue. Analisando os resultados, nota-se que após ocorrer à separação água/índigo houve uma redução significativa, comprovando a eficiência na separação, principalmente da membrana de polisulfona com 5\% de alumina, onde seu permeado se apresenta com concentrações de corante inferiores a 5 ppm. Este comportamento pode ter ocorrido devido à rejeição por exclusão de tamanho, como foi evidenciado, a partir das imagens de MEV, que estas membranas possuem uma maior quantidade de poros com diâmetros menores (Rambabu et al., 2019). Assim, provavelmente com uma maior quantidade de alumina, tem-se a tendência de melhorar a separação entre a água e o corante. 
Tabela 3 - Análise da turbidez do permeado através da quantidade das partículas suspensas após a utilização das membranas e a porcentagem de rejeição.

\begin{tabular}{cccc}
\hline Amostras & NTU & PPM & $\%$ \\
\hline PSU Pura & 1,97 & 7,04 & 97.65 \\
PSU + 3\% & 1,87 & 6,68 & 97,78 \\
$\mathrm{Al}_{2} \mathrm{O}_{3}$ & & & \\
$\mathrm{PSU}+5 \%$ & & \\
$\mathrm{Al}_{2} \mathrm{O}_{3}$ & 1,05 & 3,75 & 98,75 \\
\hline
\end{tabular}

Fonte: Autores.

\section{Considerações Finais}

Por meio dos estudos, pôde-se concluir que foi possível realizar a síntese das membranas compósitas polisulfona/alumina através do método de inversão de fase. A partir das caracterizações realizadas, verificou-se uma mudança da morfologia na seção transversal das membranas, bem como, uma correlação da quantidade de alumina com a hidrofilicidade da membrana. Além disso, com o aumento da concentração de alumina, mais poros foram gerados com diâmetros menores. Assim, pode-se correlacionar essa maior quantidade de poros com o aumento do fluxo no interior da membrana. Desta forma, com estas alterações, observou-se para o tratamento de efluentes uma diminuição da turbidez da água em torno de $98 \%$.

\section{Agradecimentos}

Os autores agradecem à UFCG pela infraestrutura, aos Laboratórios de Desenvolvimento e Caracterização de Membranas (LDCM), ao Conselho Nacional de Desenvolvimento Científico e Tecnológico - CNPq e à Coordenação de Aperfeiçoamento de Pessoal de Nível Superior - CAPES, pelo apoio financeiro.

\section{Referências}

Ahn, J., Chung, W. J., Pinnau, I., \& Guiver, M. D. (2008). Polysulfone/silica nanoparticle mixed-matrix membranes for gas separation. Journal of Membrane Science, 314(1-2), 123-133. https://doi.org/10.1016/j.memsci.2008.01.031

Alexandre, M., \& Dubois, P. (2000). Polymer-layered silicate nanocomposites: Preparation, properties and uses of a new class of materials. Materials Science and Engineering R: Reports, 28(1), 1-63. https://doi.org/10.1016/S0927-796X(00)00012-7

Anadão, P., Sato, L. F., Wiebeck, H., \& Valenzuela-Díaz, F. R. (2010). Montmorillonite as a component of polysulfone nanocomposite membranes. Applied Clay Science, 48(1-2), 127-132. https://doi.org/10.1016/j.clay.2009.12.011

Barbosa Ferreira, R. da S., Salviano, A. F., Lima Oliveira, S. S., Araújo, E. M., Medeiros, V. da N., \& Lira, H. de L. (2019). Treatment of effluents from the textile industry through polyethersulfone membranes. Water (Switzerland), 11(12). https://doi.org/10.3390/w11122540

Bertotto, R., Duarte, J., Santos, V. dos, Zeni, M., \& Bergmann, C. P. (2018). Análise do comportamento de membranas cerâmicas de $\alpha$-alumina/Pd para separação de gases a temperatura ambiente e baixas pressões. Cerâmica, 64(372), 477-484. https://doi.org/10.1590/0366-69132018643722338

Bidsorkhi, H. C., Riazi, H., Emadzadeh, D., Ghanbari, M., Matsuura, T., Lau, W. J., \& Ismail, A. F. (2016). Preparation and characterization of a novel highly hydrophilic and antifouling polysulfone/nanoporous TiO2nanocomposite membrane. Nanotechnology, 27(41). https://doi.org/10.1088/09574484/27/41/415706

Botvay, A., Máthé, Á., Pöppl, L., Rohonczy, J., \& Kubatovics, F. (1999). Preparation and characterization of brominated polyethersulfones. Journal of Applied Polymer Science, 74(1), 1-13. https://doi.org/10.1002/(SICI)1097-4628(19991003)74:1<1::AID-APP1>3.0.CO;2-7

Buruga, K., Song, H., Shang, J., Bolan, N., Jagannathan, T. K., \& Kim, K.-H. (2019). A review on functional polymer-clay based nanocomposite membranes for treatment of water. Journal of Hazardous Materials, 379, 120584. https://doi.org/10.1016/j.jhazmat.2019.04.067 
Chen, J., Shen, L., Zhang, M., Hong, H., He, Y., Liao, B. Q., \& Lin, H. (2016). Thermodynamic analysis of effects of contact angle on interfacial interactions and its implications for membrane fouling control. Bioresource Technology, 201, 245-252. https://doi.org/10.1016/j.biortech.2015.11.063

Habert, A. C., Borges, C. P., \& Nobrega, R. (2006). Processos de Separação por Membranas. In e-papers (1st ed.). Retrieved from 200.144.189.97/phd/LeArq.aspx?id_arq=4539

Homayoonfal, M., Mehrnia, M. R., Rahmani, S., \& Mohades Mojtahedi, Y. (2015). Fabrication of alumina/polysulfone nanocomposite membranes with biofouling mitigation approach in membrane bioreactors. Journal of Industrial and Engineering Chemistry, 22, 357-367. https://doi.org/10.1016/j.jiec.2014.07.031

Jamshidi Gohari, R., Lau, W. J., Matsuura, T., \& Ismail, A. F. (2013). Fabrication and characterization of novel PES/Fe-Mn binary oxide UF mixed matrix membrane for adsorptive removal of As(III) from contaminated water solution. Separation and Purification Technology, 118, 64-72. https://doi.org/10.1016/j.seppur.2013.06.043

Lenntech. (2019). Water treatment and air purification. Retrieved August 20, 2019, from https://www.lenntech.com/ceramic-membranes.htm

Maximous, N., Nakhla, G., Wan, W., \& Wong, K. (2009). Preparation, characterization and performance of Al2O3/PES membrane for wastewater filtration. Journal of Membrane Science, 341(1-2), 67-75. https://doi.org/10.1016/j.memsci.2009.05.040

Meksi, N., Kechida, M., \& Mhenni, F. (2007). Cotton dyeing by indigo with the borohydride process: Effect of some experimental conditions on indigo reduction and dyeing quality. Chemical Engineering Journal, 131(1-3), 187-193. https://doi.org/10.1016/j.cej.2007.01.001

Mulder, M. (1991). Basic Principles of Membrane Technology. https://doi.org/10.1017/CBO9781107415324.004

Nakatsuka, S., Nakate, I., \& Miyano, T. (1996). Drinking water treatment by using ultrafiltration hollow fiber membranes. Desalination, 106(1-3), 55-61. https://doi.org/10.1016/S0011-9164(96)00092-6

Palacio, L., Calvo, J. I., Prádanos, P., Hernández, A., Väisänen, P., \& Nyström, M. (1999). Contact angles and external protein adsorption onto UF membranes. Journal of Membrane Science, 152(2), 189-201. https://doi.org/10.1016/S0376-7388(98)00203-8

Rambabu, K., Bharath, G., Monash, P., Velu, S., Banat, F., Naushad, M., \& Loke Show, P. (2019). Effective treatment of dye polluted wastewater using nanoporous $\mathrm{CaCl} 2$ modified polyethersulfone membrane. Process Safety and Environmental Protection, $124, \quad 266-278$. https://doi.org/10.1016/j.psep.2019.02.015

Rodrigues, R., Mierzwa, J. C., \& Vecitis, C. D. (2019). Mixed matrix polysulfone/clay nanoparticles ultrafiltration membranes for water treatment. Journal of Water Process Engineering, 31(2), 100788. https://doi.org/10.1016/j.jwpe.2019.100788

Santos Filho, E. A. dos, Medeiros, K. M. de, Araújo, E. M., Ferreira, R. da S. B., Oliveira, S. S. L., \& Medeiros, V. da N. (2019). Membranes of polyamide 6/clay/salt for water/oil separation. Materials Research Express, 6(10), 105313. https://doi.org/10.1088/2053-1591/ab3754

Tizchang, A., Jafarzadeh, Y., Yegani, R., \& Khakpour, S. (2019). The effects of pristine and silanizes nanodiamond on the performance of polysulfone membranes for wastewater treatment by MRB system. Journal of Environmental Chemical Engineering, 7(6), 30. https://doi.org/https://doi.org/10.1016/j.jece.2019.103447

Turken, T., Sengur-Tasdemir, R., Ates-Genceli, E., Tarabara, V. V., \& Koyuncu, I. (2019). Progress on reinforced braided hollow fiber membranes in separation technologies: A review. Journal of Water Process Engineering, 32(May), 100938. https://doi.org/10.1016/j.jwpe.2019.100938

Vatanpour, V., Madaeni, S. S., Moradian, R., Zinadini, S., \& Astinchap, B. (2011). Fabrication and characterization of novel antifouling nanofiltration membrane prepared from oxidized multiwalled carbon nanotube/polyethersulfone nanocomposite. Journal of Membrane Science, 375(1-2), 284-294. https://doi.org/10.1016/j.memsci.2011.03.055

Xu, Y., Goh, K., Wang, R., \& Bae, T. H. (2019). A review on polymer-based membranes for gas-liquid membrane contacting processes: Current challenges and future direction. Separation and Purification Technology, 229(July), 115791. https://doi.org/10.1016/j.seppur.2019.115791

Yan, L., Li, Y. S., \& Xiang, C. B. (2005). Preparation of poly(vinylidene fluoride)(pvdf) ultrafiltration membrane modified by nano-sized alumina (Al2O3) and its antifouling research. Polymer, 46(18), 7701-7706. https://doi.org/10.1016/j.polymer.2005.05.155

Yuan, H. G., Liu, Y. Y., Liu, T. Y., \& Wang, X. L. (2017). Self-standing nanofilms of polysulfone doped with sulfonated polysulfone via solvent evaporation for forward osmosis. Journal of Membrane Science, 523, 567-575. https://doi.org/10.1016/j.memsci.2016.09.034 